Improving the forward solver for the complete electrode model in EIT using algebraic multigrid

Soleimani, M. and Powell, C.E. and Polydorides, N.

2005

MIMS EPrint: 2006.250

Manchester Institute for Mathematical Sciences

School of Mathematics

The University of Manchester

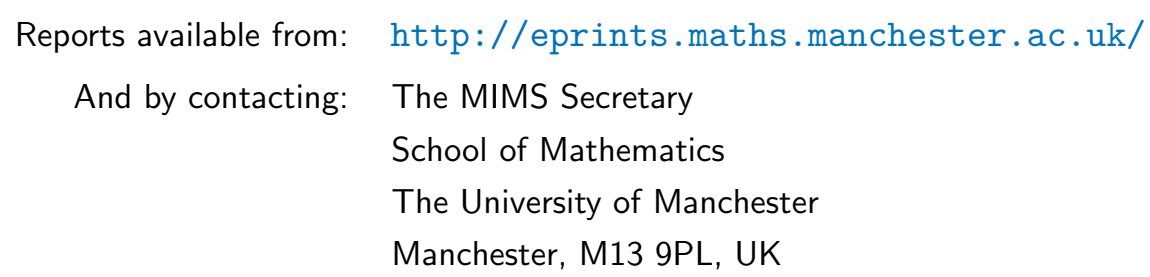

ISSN 1749-9097 


\title{
Improving the Forward Solver for the Complete Electrode Model in EIT Using Algebraic Multigrid
}

\author{
Manuchehr Soleimani, Catherine E. Powell, and Nick Polydorides*
}

\begin{abstract}
Image reconstruction in electrical impedance tomography is an ill-posed nonlinear inverse problem. Linearization techniques are widely used and require the repeated solution of a linear forward problem. To account correctly for the presence of electrodes and contact impedances, the so-called complete electrode model is applied. Implementing a standard finite element method for this particular forward problem yields a linear system that is symmetric and positive definite and solvable via the conjugate gradient method. However, preconditioners are essential for efficient convergence. Preconditioners based on incomplete factorization methods are commonly used but their performance depends on user-tuned parameters. To avoid this deficiency, we apply black-box algebraic multigrid, using standard commercial and freely available software. The suggested solution scheme dramatically reduces the time cost of solving the forward problem. Numerical results are presented using an anatomically detailed model of the human head.
\end{abstract}

Index Terms-Algebraic multigrid, complete electrode model, electrical impedance tomography, finite element method, forward problem, preconditioning.

\section{INTRODUCTION}

$\mathbf{I}$ $\mathrm{N}$ electrical impedance tomography (EIT) an image of the electrical conductivity of the interior of an object is reconstructed from measurements of electric potentials captured at its boundaries. Specifically, a set of low-frequency current patterns are driven into the domain using some boundary electrodes and potential measurements are captured on others. This technique benefits a growing number of important practical applications in the fields of industrial process monitoring and biomedical imaging. In the low-frequency cases where the conductivity coefficients are purely real, the process is known as electrical resistance tomography (ERT). Indeed, ERT has been successfully tested to detect epileptic seizures (see [4]), functional brain activity triggered by external stimuli (see [20]) and internal cortical hemorrhage (see [6]), conditions which all cause local and temporal conductivity changes in brain tissue. We do not restrict ourselves to a particular application. Rather, we focus attention, broadly, on media with conductivity distributions with coefficients that are real and possibly anisotropic and/or discontinuous.

Manuscript received March 25, 2004; revised December 27, 2004. The Associate Editor responsible for coordinating the review of this paper and recommending its publication was C. McLeod. Asterisk indicates corresponding author.

M. Soleimani and C. E. Powell are with the School of Mathematics, University of Manchester, Manchester M60 1QD, U.K.

*N. Polydorides is with the Department of Electrical and Computer Engineering, University of Cyprus, 75 Kallipoleos St., P.O. Box 20537, 1678, Nicosia, Cyprus (e-mail: nick.polydorides@ucy.ac.uk).

Digital Object Identifier 10.1109/TMI.2005.843741
Compared to more traditional techniques like X-ray computed tomography and magnetic resonance imaging (MRI), impedance imaging is conveniently portable and cost effective. However, its high demand for computational power is often regarded as a severe drawback, especially in clinical applications where on-line temporal resolution is required and large anatomically detailed models are used. The aim of this work is to improve the computational efficiency of reconstruction algorithms by accelerating their forward solvers. This is particularly useful for nonlinear schemes based on linearization, where a considerable amount of the overall reconstruction time is consumed on repetitive forward computations required for evaluating the sensitivity-Jacobian matrix [21]. However, the preconditioning scheme we describe below also benefits other reconstruction techniques based exclusively on forward computations which do not require the explicit computation of the Jacobian matrix. These include the Markov Chain Monte Carlo method (MCMC) [29], the monotonocity based algorithm [27], and the adjoint fields algorithm [8].

The focus of this paper is the fast and efficient solution of the symmetric and positive definite (SPD) linear systems, $A \underline{x}=$ $\underline{b}$, that arise from a standard finite element discretization (see [18]), of forward problems associated with the complete electrode model (see [25].) In real-life applications, where we are dealing with very complicated geometries in three space dimensions, these systems of equations are extremely large (see, for example, [7], [9], and [13].) Hence, iterative solution methods outperform direct ones. Here, the conjugate gradient method (CG) is the solver of choice, having the optimal approximation property that the iterate $\underline{x}^{i}$ produced at the $i$ th step, has minimal error $\underline{e}^{i}=\underline{x}-\underline{x}^{i}$, measured in the norm

$$
\left\|\underline{e}^{i}\right\|_{A}^{2}=\underline{e}^{i^{T}} A \underline{e}^{i}
$$

over all possible choices of $\underline{x}^{i}$ belonging to the associated Krylov subspace, (see, for example, [12] or [11].) Convergence of the method is completely determined by the spread of the eigenvalues of the coefficient matrix, in that

$$
\frac{\left\|\underline{e}^{i}\right\|_{A}}{\left\|\underline{e}^{0}\right\|_{A}} \leq \min _{p_{i}} \max _{j=1: n}\left|p_{i}\left(\lambda_{j}\right)\right|
$$

(see [12]) where $p_{i}$ is any polynomial of degree $i$ satisfying $p_{i}(0)=1$ and $\left\{\lambda_{j}\right\}_{j=1}^{n}$ denotes the set of eigenvalues of $A \in$ $\mathbb{R}^{n \times n}$. Simply put, the error on the left-hand side of (1) can only be reduced to zero with a few iterations of CG if the eigenvalues of $A$ are clustered. As is common in finite element problems, the SPD matrix $A$ in our study does not have this property. Efficient CG convergence can only be achieved by locating a symmetric preconditioner $P \in \mathbb{R}^{n \times n}$, such that the eigenvalues 
of $P^{-1} A$ are clustered, and iterating, instead, on the preconditioned system

$$
P^{-1} A \underline{x}=P^{-1} \underline{b} .
$$

The complete electrode model is also studied in [20]. The authors of that work apply incomplete Cholesky (IC) factorization to precondition the forward problem. One disadvantage with this approach is that the user does not know a priori how much fill-in to allow. Complete factorizations may yield optimal convergence rates but the time cost for factorization is totally unacceptable. Some trade-off has to be made between the permitted level of fill-in and the convergence properties of CG for the resulting preconditioned system. The upshot is that the user has to experiment in order to determine the optimal drop tolerance parameter. This is too time-consuming.

In [14], geometric multigrid (GMG) is considered as an alternative solver for a similar forward problem in electrical impedance tomography. Whilst using GMG to solve such problems on simple closed geometries such as cubes or other regular polyhedrons is relatively straightforward, this is not so for complicated domains (such as the human head, see [1], [10]) with discontinuous and/or anisotropic coefficients. In particular, it requires the generation of a hierarchy of finite element meshes which can be vastly expensive and time-consuming.

Algebraic multigrid, or AMG, (see [23], [24], and [26]) is a highly attractive plug-in solver for three-dimensional (3-D) problems posed on irregular domains. Popularized by Ruge and Stüben in the 1980s, the approach is derived from traditional multigrid principles (see [2]), but, crucially, does not require the user to supply geometric information associated with a hierarchy of finite element meshes. Convergence theory is largely based on heuristic arguments and limited to the class of so-called M-matrices. However, the resulting scheme has optimal work complexity and provided that basic criteria are satisfied, it can be applied as a black-box preconditioner, i.e., without tuning parameters.

In [17] and [16], forward problems with different boundary conditions are studied. The first work reports on the efficiency of AMG as a solver for a finite difference discretization, which does yield an M-matrix. In the second, a finite element method is applied and the resulting system is not an M-matrix. The authors recommend that $\mathrm{AMG}$ is applied to a spectrally equivalent M-matrix and used as a preconditioner for the original system. The system matrix for the forward problem in the complete electrode model, to be discussed later, has a slightly different structure. This is the first time that AMG has been investigated as a solution methodology for it. Our numerical experience reveals that AMG can be applied effectively as a black-box preconditioner for the original system matrix despite the presence of positive off-diagonal entries which violate the M-matrix property.

The paper is organized as follows. In Section II we review the complete electrode model. Section III describes properties of the associated algebraic system. In Sections IV and V we discuss AMG and preconditioning. Numerical results, including a case study with a 3-D head model, are presented in Sections VI and VII.

\section{COMPLETE Electrode Model}

Let $\Omega \subset \mathbb{R}^{3}$ be a Lipschitz domain with $C^{2}$-continuous boundary $\Gamma$, to which $L$ electrodes are attached. In the absence of interior sources or charges, the low-frequency, time-harmonic Maxwell's equations reduce to the elliptic partial differential equation

$$
\nabla \cdot(\hat{\sigma} \nabla u)=0 \text { in } \Omega .
$$

Here, $u$ is the scalar electrical potential and $\hat{\sigma}=\hat{\sigma}(\vec{x})$ is a symmetric and positive definite tensor of electrical conductivity coefficients. Equation (2) is solved in conjunction with the set of boundary conditions prescribed by the so-called complete electrode model. A theoretical study of the model can be found in [25] and details of finite element implementations and numerical considerations are given in [28] and [21]. Specifically, the boundary current density satisfies

$$
\begin{array}{ccc}
\int_{e_{l}} \hat{\sigma} \nabla u \cdot \hat{n}=I_{l} & \text { on } \quad \Gamma_{1} \\
\hat{\sigma} \nabla u \cdot \hat{n}=0 & \text { on } \quad \Gamma_{2}
\end{array}
$$

while for the boundary electric potential measurements, the relation

$$
u+z_{l} \hat{\sigma} \nabla u \cdot \hat{n}=V_{l} \quad \text { on } \quad \Gamma_{1}
$$

is valid. Here, $I_{l}$ denotes the current on the surface of the $l$ th electrode, $e_{l}, V_{l}$ is the electric potential measured by $e_{l}, z_{l}$ is the associated contact impedance and $\hat{n}$ is the outward-pointing unit normal vector. In addition, $\Gamma_{1} \subset \Gamma$ denotes the union of the pieces of the boundary situated underneath the electrodes and $\Gamma_{2}=\Gamma \backslash \Gamma_{1}$ is the remainder of the surface. The model is known (see [25]) to have a unique solution up to an additive constant. Hence, one can apply a reference condition for the potential by grounding one of the boundary electrodes, yielding the Dirichlet boundary condition

$$
u=\left.0\right|_{\Gamma_{G}} \quad \Gamma_{G} \subset \Gamma_{1} .
$$

Alteratively, (6) can be applied to a randomly selected node in the model. Applying the charge conservation theorem, we also impose

$$
\sum_{l=1}^{L} I_{l}=0 .
$$

\section{LiNEAR Algebra PRoBlem}

To solve (2) together with (3)-(5) numerically, the domain is partitioned into $k$ tetrahedra with a total of $n$ vertices. The conductivity coefficients are each approximated by a piecewise constant function on that mesh. Given a standard nodal basis $\left\{\phi_{i}\right\}_{i=1}^{n}$ for the set of piecewise linear functions, a potential is sought in the form

$$
u_{h}=\sum_{i=1}^{n} u_{i} \phi_{i} .
$$


Multiplying (2) by an arbitrary test function $v$, which is sufficiently smooth, and integrating over $\Omega$ gives

$$
\int_{\Omega} v \nabla \cdot\left(\hat{\sigma} \nabla u_{h}\right) d V=0 \quad \text { in } \Omega .
$$

Integrating by parts yields

$$
\int_{\Omega} \hat{\sigma} \nabla u_{h} \cdot \nabla v d V=\int_{\Gamma_{1}} \hat{\sigma} \nabla u_{h} \cdot \hat{n} v d S+\int_{\Gamma_{2}} \hat{\sigma} \nabla u_{h} \cdot \hat{n} v d S
$$

so that imposing (4) and (5) on $u_{h}$ gives

$$
\int_{\Omega} \hat{\sigma} \nabla u_{h} \cdot \nabla v d V=\sum_{l=1}^{L} \int_{e_{l}} \frac{1}{z_{l}}\left(V_{l}-u_{h}\right) v d S .
$$

Substituting for $u_{h}$ from (8) in (10) gives

$$
\begin{aligned}
\sum_{i=1}^{n}\left\{\left(\int_{\Omega} \hat{\sigma} \nabla \phi_{i} \cdot \nabla v d V+\right.\right. & \left.\left.\sum_{l=1}^{L} \int_{e_{l}} \frac{1}{z_{l}} \phi_{i} v d S\right)\right\} u_{i} \\
& -\sum_{l=1}^{L}\left(\int_{e_{l}} \frac{1}{z_{l}} v d S\right) V_{l}=0
\end{aligned}
$$

so that setting $v=\phi_{j}, j=1: n$, yields $n$ algebraic equations. Imposing the remaining boundary condition (3) on $u_{h}$ and applying (5) yields an additional $L$ algebraic equations

$$
I_{l}=\frac{1}{z_{l}} V_{l}\left|e_{l}\right|-\sum_{i=1}^{n}\left(\int_{e_{l}} \frac{1}{z_{l}} \phi_{i} d S\right) u_{i}, \quad l=1: L
$$

where, here, $\left|e_{l}\right|$ denotes the area of the $l$ th electrode. Hence, assembling $A_{M} \in \mathbb{R}^{n \times n}, A_{Z} \in \mathbb{R}^{n \times n}, A_{V} \in \mathbb{R}^{n \times L}$, and $A_{D} \in \mathbb{R}^{L \times L}$, via

$$
\begin{aligned}
& A_{M}(i, j)=\int_{\Omega} \hat{\sigma} \nabla \phi_{i} \cdot \nabla \phi_{j} d V \quad i, j=1: n, \\
& A_{Z}(i, j)=\sum_{l=1}^{L} \int_{e_{l}} \frac{1}{z_{l}} \phi_{i} \phi_{j} d S \quad i, j=1: n, \\
& A_{V}(i, l)=-\int_{e_{l}} \frac{1}{z_{l}} \phi_{i} d S \\
& A_{D}(s, l)=\left\{\begin{array}{cc}
\frac{1}{z_{l}}\left|e_{l}\right| & s=l \\
0 & s \neq l
\end{array}, \quad s, l=1: L, l=1: L,\right.
\end{aligned}
$$

leads to the matrix equation

$$
\left(\begin{array}{cc}
A_{M}+A_{Z} & A_{V} \\
A_{V}^{T} & A_{D}
\end{array}\right)(\underline{\underline{u}} \underline{\underline{v}})=\left(\frac{\underline{0}}{\underline{I}}\right)
$$

where $\underline{u}(i)=u_{i}, \underline{v}(l)=V_{l}$, and $\underline{I}(l)=I_{l}$, for $i=1: n$ and $l=1: L$.

\begin{tabular}{|c|c|c|}
\hline Level 1 nodes: & $\Omega_{1}=1: N$ & \\
\hline Splitting routine: & $\Omega_{1} \rightarrow C \cup F$ & \\
\hline Level 2 nodes: & $\Omega_{2}=C \subset \Omega_{1}$ & \\
\hline Smoother: & $\mathcal{S}=$ Gauss-Seidel & \\
\hline Interpolation: & $\left(I_{2}^{1} \underline{v}^{2}\right)_{i}=\left\{\begin{array}{c}\underline{v}_{i}^{2} \\
\sum_{j \in P_{i}} \omega_{i j} \underline{v}_{j}^{2}\end{array}\right.$ & $\begin{array}{l}\text { if } i \in C \\
\text { if } i \in F\end{array}$ \\
\hline Restriction: & $I_{1}^{2}=\left(I_{2}^{1}\right)^{T}$ & \\
\hline Level 2 matrix: & $A^{2}=I_{1}^{2} A^{1} I_{2}^{1}$ & \\
\hline
\end{tabular}

Attention must be paid to the efficiency of the solution of (13) since it must be solved for a number of right-hand sides, corresponding to different current patterns. For brevity, we write the system (13) as $A \underline{x}=\underline{b}$. Note that since the problem is well-posed (see [25]), $A$ is SPD. We employ CG as a solver and use a single V-cycle of AMG as a preconditioner. Since the contact impedances and the averaged conductivity coefficients are positive, it follows immediately from the above definitions
TABLE I

AMG COMPONENTS

and the definition of the standard linear basis functions (e.g., see [5]) that:

- $A_{M}(i, i)>0, A_{Z}(i, i)>0$, for $i=1: n$;

- $A_{D}(l, l)>0$ for $l=1: L$;

- $A_{Z}(i, j) \geq 0$ for $i, j=1: n$;

- $A_{V}(i, l) \leq 0$ for $i=1: n, j=1: L$.

All these properties have consequences for AMG. Note that since $L \ll n, A_{V}$ and $A_{D}$ represent only a few rows and columns of the whole coefficient matrix. The success of any multigrid preconditioner is thus determined by the properties of $A_{M}+A_{Z}$. Here, $A_{Z}$ and $A_{M}$ contribute positive off-diagonal entries to $A$, violating the M-matrix property.

Definition 1: An SPD matrix $A \in \mathbb{R}^{n \times n}$ is an M-matrix if:

- $A(i, i)>0, i=1: n$;

- $A(i, j) \leq 0, i, j=1: n, i \neq j$.

However, $A_{Z}$ is extremely sparse if the number of electrodes is small. In practice, the number of significant positive off-diagonal entries contributed by $A_{Z}$ is small.

\section{Algebraic MultigRID}

AMG embodies a family of methods for solving sparse linear systems. The original algorithm amg1r5, designed for SPD M-matrices, was developed in the 1980s (see [23]). Extensions to more general problems have been introduced (e.g., see [26]) but freely available source codes are mainly based on the original algorithm. Although convergence theory is limited to M-matrices, AMG has two crucial benefits for tackling 3-D problems. Work complexity depends linearly on the problem size and no geometric information is required.

Let $A^{1} \underline{x}^{1}=\underline{b}^{1}$ denote the linear system of size $N=n+L$ to be solved. We refer to this as the 'level-one' problem. As in geometric multigrid, given an initial guess $\underline{x}^{1,0}$, we attempt to reduce error by combining smoothing and coarse-level correction. The basic components of a two-level method for M-matrices are listed in Table I.

In AMG, the smoother $\mathcal{S}$ is a simple stationary iterative method such as Gauss-Seidel or Jacobi iteration. Coarse level nodes, $C$, are just subsets of the original unknowns $\Omega_{1}=1: N$. Interpolation and restriction matrices $I_{2}^{1}$ and $I_{1}^{2}$ are needed to communicate level-two vectors $\underline{v}^{2}$ and level-one vectors $\underline{v}^{1}$ between levels. To interpolate a level-two vector $\underline{v}^{2}$ to level 
TABLE II

Two LEVEL V-CyCLE WITH $\nu$ SMOOTHING STEPS

\begin{tabular}{ll}
\hline Pre-smooth: & $\underline{x}^{1,1}=S^{\nu}\left(\underline{x}^{1,0}, A^{1}, \underline{b}^{1}\right)$ \\
Compute residual : & $\underline{r}^{1}=\underline{b}^{1}-A^{1} \underline{x}^{1,1}$ \\
Restrict: & $\underline{r}^{2}=I_{1}^{2} \underline{r}^{1}$ \\
Solve exactly : & $A^{2} \underline{e}^{2}=\underline{r}^{2}$ \\
Interpolate: & $\underline{e}^{1}=I_{2}^{1} \underline{e}^{2}$ \\
Correct: & $\underline{x}^{1,2}=\underline{x}^{1,1}+\underline{e}^{1}$ \\
Post-smooth : & $\underline{x}^{1,3}=S^{\nu}\left(\underline{x}^{1,2}, A^{1}, \underline{b}^{1}\right)$ \\
\hline
\end{tabular}

one, values at C-points are simply injected. The value at an F-point is taken as a weighted linear combination of values at neighboring C-points. Hence, a set of interpolation nodes $P_{i}$ is required together with a set of weights $\left\{w_{i, j}\right\}$. The operation $(\cdot)^{T}$ denotes matrix transposition.

The basic two level V-cycle method, with $\nu$ smoothing steps is summarized in Table II. Here, $S^{\nu}\left(\underline{x}^{1, j}, A^{1}, \underline{b}^{1}\right), j=0,2$, denotes the application, $\nu$ times, of the chosen smoother to the linear system $A^{1} \underline{x}^{1, j}=\underline{b}^{1}$. To define a multilevel scheme, the system $A^{2} \underline{e}^{2}=\underline{r}^{2}$ is now solved approximately. This is obtained by recursively calling the code, cycling down to a level consisting of a very few nodes. For M-matrices it is easy to derive algebraic equations for the error that cannot be reduced by Gauss-Seidel smoothing (see [3]). The coarse levels, interpolation variables $P_{i}$ and weights $\left\{\omega_{i j}\right\}$ are chosen to capture that error. The standard choices are derived as follows.

Definition 2: If $A^{J}$ is an M-matrix, a node $j$ is strongly connected to $i$, relative to $\theta$ with $0<\theta<1$, if

$$
-A_{i j}^{J} \geq \theta \max _{k \neq i}\left\{-A_{i k}^{J}\right\}
$$

We use the default value $\theta=0.25$. At level $\mathrm{J}$, we find for each node $i=1: N_{J}$, its strongly connected neighbors, $S_{i}$, and perform an initial $\mathrm{C} / \mathrm{F}$ (coarse-fine) splitting using the algorithm shown in Table III. Step two says that ranks, $R_{i}$, are assigned to each undecided node $i \in U$ by counting how many other nodes $j$ strongly depend on it. In the remaining steps, nodes with the largest ranks are chosen as coarse level (C) nodes as they are the most desirable interpolation variables. In a second phase, if any strong F-F connections do not share an interpolation $\mathrm{C}$-node, some $\mathrm{F}$-nodes are changed to $\mathrm{C}$-nodes.

Now, at any level, $J$, given subsets $C$ and $F$, we distinguish, for nodes $i=1: N_{J}$, three types of connections in the row $A^{J}(i,:)$. These are

$$
C_{i}^{s}=S_{i} \cap C, \quad D_{i}^{s}=S_{i} \cap F, \quad \text { and } \quad D_{i}^{w}
$$

strong C-nodes, strong F-nodes and weakly connected nodes. For M-matrices it can be shown that error not eliminated by Gauss-Seidel relaxation satisfies

$$
A_{i i}^{J} \underline{e}_{i}^{J} \approx-\sum_{j \in C_{i}^{s}} A_{i j}^{J} \underline{e}_{j}^{J}-\sum_{j \in D_{i}^{s}} A_{i j}^{J} \underline{e}_{j}^{J}-\sum_{j \in D_{i}^{w}} A_{i j}^{J} \underline{e}_{j}^{J} .
$$

To determine $\underline{e}_{i}^{J}$ as a linear combination of errors at neighboring points we choose interpolation nodes $P_{i}=C_{i}^{S}$ and approximate
TABLE III

COARSENING: PHASE I

\begin{tabular}{ll}
\hline 1. & set $C=F=\emptyset$ and $U=1: N_{J}$, \\
2. & $\forall i \in U$, set $S_{i}^{T}=\left\{j \mid i \in S_{j}\right\}$ and $R_{i}=\left|S_{i}^{T}\right|$, \\
3. for $i$ with max. $R_{i}$ set $C=C \cup\{i\}, U=U-\{i\}$, \\
4. $\forall j \in S_{i}^{T} \cap U$, set $F=F \cup\{j\}$ and $U=U-\{j\}$, \\
5. $\forall k \in S_{j}^{T} \cap U$, set $R_{k}=R_{k}+1$, \\
6. $\forall j \in S_{i}^{T} \cap U$, set $R_{j}=R_{j}-1$, \\
7. $\quad$ Return to 3. and continue until $U=\emptyset$.
\end{tabular}

$\underline{e}_{j}^{J}$ for $j \in D_{i}^{s} \cup D_{i}^{w}$ using the error at nodes $i$ and $j \in P_{i}$. Using standard techniques, one can derive a simple interpolation scheme as outlined in Table I with weights

$$
w_{i j}=-\frac{a_{i j}+\sum_{m \in D_{i}^{s}}\left(\frac{a_{i m} a_{m j}}{\sum_{k \in C_{i}^{s}} a_{m k}}\right)}{a_{i i}+\sum_{n \in D_{i}^{w}} a_{i n}} .
$$

Full details of the algorithm can be found in [23].

\section{PRECONDITIONING}

The above method, due to Ruge and Stüben, only provides a robust solver for systems whose coefficient matrices are M-matrices (see [23] and [24]). The matrix $A$ in (13) does not belong to this class. However, since the M-matrix property is not violated too strongly, the basic AMG algorithm designed for M-matrices still provides an excellent preconditioner for the Krylov subspace solver CG. Here, CG is the solver of choice because it has an optimal approximation property for SPD systems. In each CG iteration, when the action of a preconditioner is required, we simply apply one $\mathrm{V}$-cycle of AMG to $A$. As long as $P^{-1}$, the matrix corresponding to the action of that $\mathrm{V}$-cycle, is symmetric then convergence is determined by the spread of the eigenvalues of $P^{-1} A$.

A problem for nonspecialists is that some commercial AMG routines do not provide symmetric preconditioners. Given an SPD matrix $A^{J}$, decomposed into its diagonal and triangular parts, $A^{J}=D+L+U$, the Gauss-Seidel relaxation matrix, $\mathcal{S}_{G S}=(D+L)$, is not symmetric. If AMG source code is available, a safe way of 'symmetrising' a V-cycle is to use the symmetric Gauss-Seidel smoother, $\mathcal{S}_{S G S}=\mathcal{S}_{G S} D^{-1} \mathcal{S}_{G S}^{T}$, which is implemented by adding, after each standard Gauss-Seidel preand post- smoothing step, a second iteration sweeping through the nodes in reverse order.

To illustrate the efficiency of the preconditioning scheme, consider a simple test problem on a cylindrical domain with anisotropic $\hat{\sigma}=\operatorname{diag}(1,2,3)$ and $z_{l}=10, l=1: 4$. The eigenvalues of $P^{-1} A$ assembled on a mesh of 1,060 nodes, where $P^{-1}$ is one V-cycle of the code amg1r5 (see [23]) with symmetric smoothing, are shown in Fig. 1. They lie in the interval $[0.7043,1]$ and consequently, only 10 iterations of CG-AMG are required to reduce the relative residual error to $10^{-8}$. When the mesh is refined, the eigenvalues do not spread out and the iteration count does not increase. 


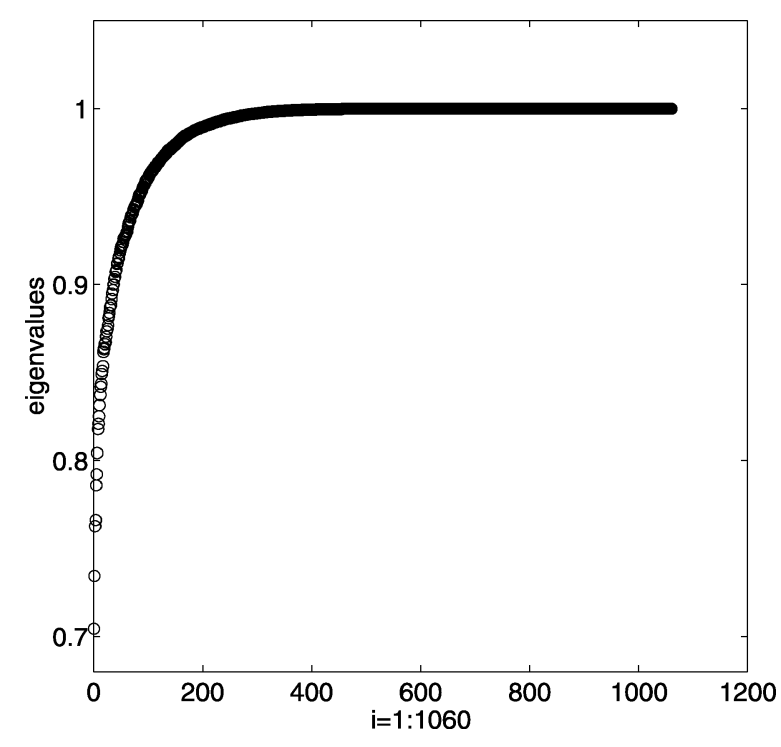

Fig. 1. Eigenvalues of $P^{-1} A ; P^{-1}$ is one V-cycle of AMG.

\section{CYLINDER TEST EXAMPLE}

In ERT applications, the time cost of solving (13) is our highest consideration. To illustrate the real benefits of using the suggested solution scheme, we present numerical results using an optimized commercial code. All the experiments in the remaining sections were performed on a Unix machine in MATLAB using FEMLAB (see [15]) and its integrated AMG software. For $C G$, we use a zero initial guess and terminate the iteration when the residual error satisfies

$$
{\sqrt{\underline{r}^{i}}}^{T} \underline{r}^{i}<10^{-8}{\sqrt{\underline{r}^{0}}}^{T} \underline{r}^{0} .
$$

To test the performance of the preconditioner with respect to mesh size, with fixed conductivity distribution, we repeat the experiment on the cylinder. Iterations counts and solution times (in elapsed seconds) for unpreconditioned CG are listed in Table IV. Results with AMG preconditioning are given in Table V.

Here, "setup" refers to the initial process of assembling the components listed in Table I. It is performed once, outside the CG iteration, so the total time cost in Table $\mathrm{V}$ is the sum of the last two columns.

In Table VI we list, for a fixed problem size, iteration counts for CG iteration with incomplete Cholesky preconditioning. This scheme is popular amongst practitioners. Here, $\epsilon$ denotes the drop tolerance parameter. The results illustrate the fact that better convergence is obtained by allowing more fill-in. However, factorization time increases unacceptably. A disadvantage for nonspecialists is that $\epsilon$ needs to be tuned to the problem at hand. One does not know, a priori which value of $\epsilon$ will yield the quickest solution time. In this example, doing no preconditioning at all is actually faster than performing the factorization.

Convergence rates of both preconditioning schemes are compared in Fig. 2. Note that in the AMG experiment, no parameters are tuned. It is applied as a black-box. The key observations are that the time cost grows linearly with respect to the problem size and the convergence rate is optimal.
TABLE IV

UNPRECONDITIONED CG, CYLINDER EXAMPLE

\begin{tabular}{rrr}
\hline$n$ & iter & solve (sec.) \\
\hline 1,060 & 178 & 0.21 \\
10,441 & 344 & 5.08 \\
93,209 & 724 & 149.90 \\
\hline
\end{tabular}

TABLE V

CG-AMG ITERATION, CYlinder EXAMPlE

\begin{tabular}{rrrr}
\hline$n$ & iter & set-up (sec.) & solve (sec.) \\
\hline 1,060 & 10 & 0.03 & 0.11 \\
10,441 & 14 & 0.31 & 1.98 \\
93,209 & 14 & 20.60 & 13.60 \\
\hline
\end{tabular}

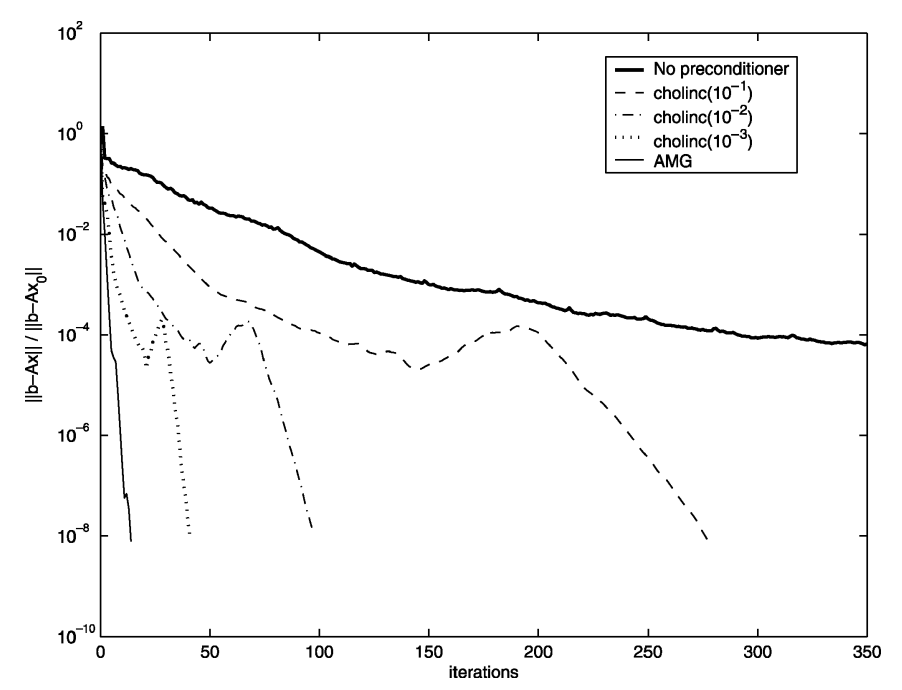

Fig. 2. Convergence of CG with $\mathrm{AMG}$ and $\mathrm{IC}$ preconditioning.

TABLE VI

CG-ChOLINC ITERATION, CYLINDER EXAMPLE, $\boldsymbol{n}=93209$

\begin{tabular}{rrrr}
\hline$\epsilon$ & iter & fac. (sec.) & solve (sec.) \\
\hline $10^{-1}$ & 396 & 3.3 & 196.75 \\
$10^{-2}$ & 97 & 262.4 & 67.70 \\
$10^{-3}$ & 41 & $5,617.0$ & 42.30 \\
\hline
\end{tabular}

\section{Cryosurgery Monitoring in A 3-D HeAd Model}

In this section, we examine the efficiency of the proposed preconditioning scheme for a challenging real-life application, involving a complicated geometry. Specifically, we are interested in the use of ERT for monitoring cryosurgery (see [19]), a technique that uses freezing to destroy tumorous tissues. We focus on the human head which contains tissues with highly discontinuous conductivity coefficients.

In this simulation, we are interested in evaluating changes in voltage measurements induced by the introduction of inhomogeneities with radically lower conductivity, (representing frozen tissue) into the brain (see [19] and [22]). We solve the complete electrode forward problem on two finite element meshes, labeled M1 and M2. The first has 9063 nodes and 44304 elements (also used in [20]) and the finest one has 59372 nodes and 327015 elements. To begin, we solve 


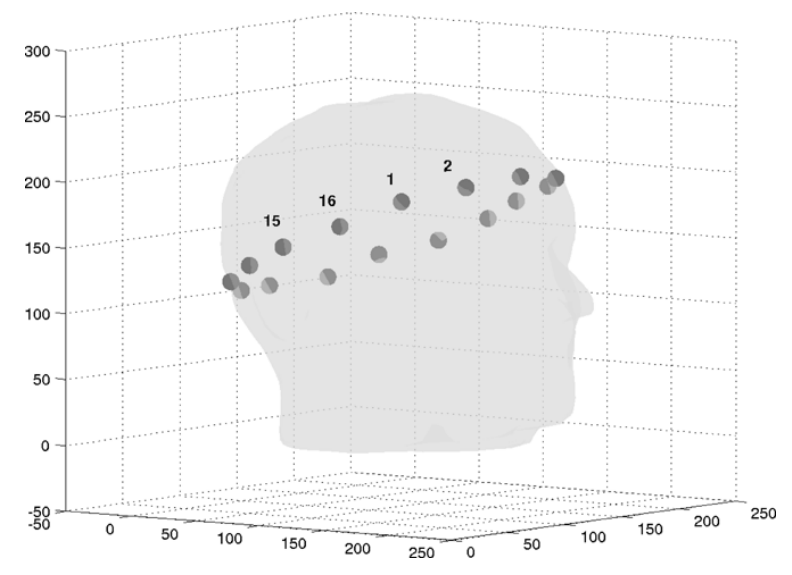

Fig. 3. Arrangement of 16 surface electrodes, numbered anti-clockwise.

the problem with the conductivity coefficients prescribed in Table VII. Next, we introduce into the domain, a spherical inhomogeneity with conductivity $10^{-5} \mathrm{Sm}^{-1}$, and diameter 15 $\mathrm{mm}$. We label these models, on meshes M1 and M2, MP1, and MP2, respectively. In all the experiments, a total of 16 electrodes are used and opposite current patterns of $1 \mathrm{~mA}$ are applied. The arrangement of the surface electrodes is shown in Fig. 3 and the area of frozen tissue is centered at the point $(100 \mathrm{~cm}, 100 \mathrm{~cm}, 100 \mathrm{~cm})$.

Table VIII summarizes the performance of CG with incomplete Cholesky preconditioning for the forward problems constructed on both meshes, with and without frozen tissue. Again, the choice of the drop tolerance $\epsilon$ for the factorization plays a key role in the time cost of each solve. Note however, that since for each forward problem one needs to solve the same linear system of equations with several right-hand sides (different current patterns), this complicates the choice of $\epsilon$. The results presented below are averaged over 15 different right-hand sides. Overall, the choice $\epsilon=10^{-3}$ proved most efficient. However, the optimal choice is hard to ascertain and depends on each individual problem.

Table IX shows the performance of CG with AMG preconditioning for the same experiments. Comparing the time costs of the two approaches, we see that the CG-AMG method is significantly faster.

Finally, we increase the volume of the frozen tissue from zero to a maximum volume of $1 \mathrm{~cm}^{3}$, in twelve steps, and evaluate the measurement voltage during the freezing process. In this case we use mesh M2. In the first step the initial guess for the $\mathrm{CG}$ iteration to solve the forward problem is set to zero. In each of the subsequent eleven steps we use the solution of the forward problem from the previous step as an initial guess. This also helps to reduce the total time cost of solving the systems. The time needed to solve the first forward problem (one current pattern) is $19.26 \mathrm{~s}$. The average time for each of the eleven remaining forward problems is only $8.36 \mathrm{~s}$.

Fig. 4 shows that the voltage difference between electrodes 1 and 8 (see Fig. 3) when the current source and sink electrodes are numbers 3 and 10. The voltage difference increases almost linearly with the volume of the frozen tissue. A similar observation has been reported in [22]. The measurement voltages for the other electrodes exhibit the same behavior.
TABLE VII

CONDUCTIVITY COEFFICIENTS FOR THE HEAD MODEL IN $\mathrm{Sm}^{-1}$

\begin{tabular}{rr}
\hline tissue & conductivity \\
\hline scalp & 0.172 \\
skull & 0.067 \\
csf & 1.540 \\
gray matter & 0.345 \\
brain & 0.150 \\
\hline
\end{tabular}

TABLE VIII

CG-IC ITERATION, DROP TOLERANCE $\epsilon=10^{-3}$

\begin{tabular}{rrrr}
\hline & iter & fac. (sec.) & solve (sec.) \\
\hline M1 & 849 & 12.21 & 45.31 \\
MP1 & 851 & 12.04 & 45.62 \\
M2 & 2,856 & 430.25 & $1,305.68$ \\
MP2 & 3,100 & 420.91 & $1,411.32$ \\
\hline
\end{tabular}

TABLE IX

CG-AMG ITERATION

\begin{tabular}{rrrr}
\hline & iter & set-up (sec.) & solve (sec.) \\
\hline M1 & 16 & 0.36 & 1.81 \\
MP1 & 18 & 0.36 & 2.01 \\
M2 & 20 & 6.06 & 12.90 \\
MP2 & 21 & 6.06 & 13.46 \\
\hline
\end{tabular}

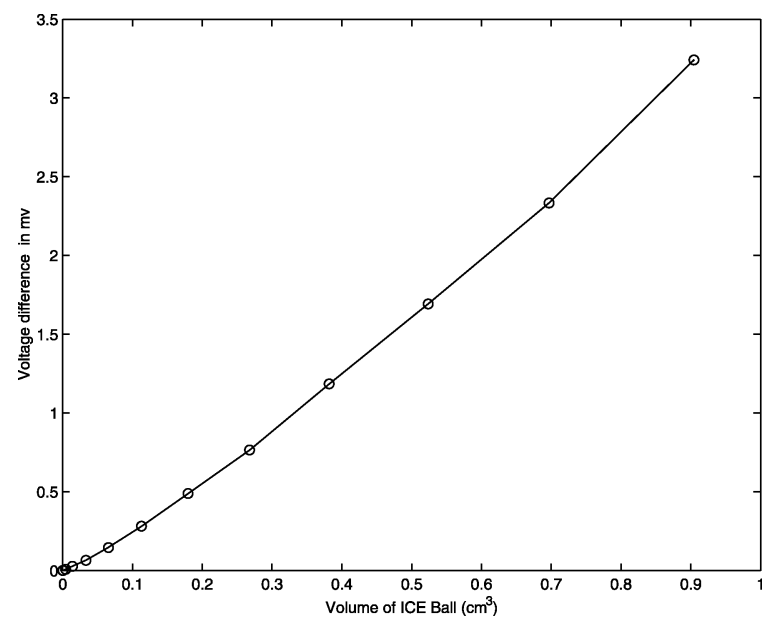

Fig. 4. Voltage differences with respect to the volume of the ice ball.

\section{CONCLUSION}

We have described the application of AMG as a black-box preconditioner for the complete electrode model, a forward problem arising in ERT applications. Numerical results illustrate that with this preconditioning scheme, the convergence of $\mathrm{CG}$ is independent of the mesh size and highly robust with respect to jumps in conductivity coefficients. Further, it offers significant advantages over traditional incomplete factorization methods. Work complexity is optimal with respect to the problem size and no parameters require tuning. As a case study we considered the feasibility of using ERT for cryosurgery monitoring. The use of AMG as a preconditioner for the forward problem leads to a significant decrease in the time cost of solving the image reconstruction problem. 


\section{ACKNOWLEDGMENT}

The authors would like to thank the SCI Institute at the University of Utah for providing the head models.

\section{REFERENCES}

[1] R. H. Bayford, A. Gibson, A. Tizzard, A. T. Tidswell, and D. S. Holder, "Solving the forward problem for the human head using IDEAS (Integrated Design Engineering Analysis Software) a finite element modeling tool," Physiological Meas., vol. 22, no. 1, pp. 55-63, 2001.

[2] J. H. Bramble, Multigrid Methods, ser. Pitman Research Notes in Mathematics, 294. White Plains, NY: Longman, 1993.

[3] W. L. Briggs, V. E. Henson, and S. F. McCormick, A Multigrid Tutorial. Philadelphia, PA: SIAM, 2000.

[4] K. Boone, A. M. Lewis, and D. S. Holder, "Imaging of cortical spreading depression by EIT: implications for localization of epileptic foci," Physiological Meas., vol. 15, pp. A189-A198, 1994.

[5] M. Cheney, D. Isaacson, J. C. Newell, S. Simske, and J. Goble, "NOSER: an algorithm for solving the inverse conductivity problem," Int. J. Imag. Syst. Technol., vol. 2, pp. 66-75, 1990.

[6] M. T. Clay and T. C. Ferree, "Weighted regularization in electrical impedance tomography with applications to acute cerebral stroke," IEEE Trans. Med. Imag., vol. 21, no. 6, pp. 629-637, Jun. 2002.

[7] A. Dey and H. F. Morrison, "Resistivity modeling for arbitrarily shaped three-dimensional structures," Geophysics, vol. 44, pp. 753-780, 1979.

[8] O. Dorn, H. Bertete-Aguirre, J. G. Berryman, and G. C. Papanicolaou, "A nonlinear inversion method for 3D electromagnetic imaging using adjoint fields," Inverse Prob., vol. 15, no. 6, pp. 1523-1558, 1999.

[9] R. G. Ellis and D. W. Oldenburg, "The pole-pole 3D DC resistivity inverse problem: a conjugate gradient approach," Geophysical J. Int., vol. 119, pp. 187-194, 1994.

[10] A. Gibson, "Electrical impedance tomography of human brain function," $\mathrm{Ph}$.D. thesis, Univ. College London, London, U.K., 2000.

[11] G. H. Golub and C. F. Van Loan, Matrix Computations. Baltimore, MD: John Hopkins Univ. Press, 1989.

[12] A. Greenbaum, Iterative Methods for Solving Linear Systems. Philadelphia, PA: SIAM, 1997.

[13] B. D. Grieve, J. L. Davidson, R. Mann, W. R. B. Lionheart, and T. A. York, "Process compliant electrical impedance instrumentation for wide scale exploitation on industrial vessels," in Proc. 3rd World Congr. Industrial Process Tomography, Banff, AB, Canada, Sep. 2-5, 2003, pp. 806-812.

[14] K. Hollaus, B. Weiss, C. H. Magele, and H. Hutten, "Geometric multigrid to accelerate the solution of the quasistatic electric field problem by tetrahedral finite elements," Physiological Meas., vol. 25, no. 1, pp. $169-179,2004$
[15] (2005) FEMLAB. Comsol, Inc., Burlington, MA. [Online] Available: http://www.comsol.com

[16] M. Kaltenbacher, S. Reitzinger, and B. Vanrumste, "Algebraic Multigrid Method for Solving 3D Nonlinear Electrostatic and Magnetostatic Field Problems," SFB F013, Linz, Österreich, Austria, Tech. Rep.00-07, Apr. 2000.

[17] M. Mohr and B. Vanrumste, "Comparing iterative solvers for linear systems associated with the finite difference discretization of the forward problem in electro-encephalographic source analysis," Med. Biol. Eng. Comput., vol. 41, no. 1, pp. 75-84, 2003.

[18] T. Murai and Y. Kagawa, "Electrical impedance tomography based on a finite element method," IEEE Trans. Biomed. Eng., vol. BME-32, pp. 177-184, 1985.

[19] D. M. Otten and B. Rubinsky, "Cryosurgical monitoring using bioimpedance measurements-a feasibility study for electrical impedance tomography," IEEE Trans. Biomed. Eng., vol. 47, no. 10, pp. 1376-1381, Oct. 2000.

[20] N. Polydorides, W. R. B. Lionheart, and H. McCann, "Krylov subspace iterative techniques: on the detection of brain activity with electrical impedance tomography," IEEE Trans. Med. Imag., vol. 21, no. 6, pp. 596-603, Jun. 2002.

[21] N. Polydorides and W. R. B. Lionheart, "A MATLAB based toolkit for three-dimensional electrical impedance tomography: a contribution to the EIDORS project," Meas. Sci. Technol., vol. 13, no. 12, pp. 1871-1883, 2002.

[22] M. M. Radai, S. Abboud, and B. Rubinsky, "Evaluation of the impedance technique for cryosurgery in a theoretical model of the head," Cryobiology, vol. 38, no. 1, pp. 51-59, 1999.

[23] J. W. Ruge and K. Stüben, "Efficient solution of finite difference and finite element equations by algebraic multigrid (AMG)," in Multigrid Methods for Integral and Differential Equations. ser. 3, The Institute of Mathematics and Its Applications Conference, D. J. Paddon and H. Holstein, Eds. Oxford, U.K.: Clarendon Press, 1985, pp. 169-212.

[24] — "Algebraic multigrid," in Multigrid Methods, S. F. McCormick, Ed. Philidelphia, PA: SIAM, 1987, pp. 73-130.

[25] E. Somersalo, D. Isaacson, and M. Cheney, "Existence and uniquenes for a electrode models for electric current computed tomography," SIAM J. Appl. Math., vol. 52, pp. 1023-1040, 1992.

[26] K. Stüben, "Algebraic Multigrid (AMG): An Introduction With Applications," in Multigrid, U. Trotenberg, C. W. Oosterlee, and A. Schüller, Eds. New York: Academic, 2000.

[27] A. Tamburrino and G. Rubinacci, "A new noniterative inversion method in electrical resistance tomography," Inverse Prob., vol. 18, pp. 1809-1829, 2002.

[28] P. J. Vauhkonen, M. Vauhkonen, T. Savolainen, and J. P. Kaipio, "Three dimensional electrical impedance tomography based on the complete electrode model," IEEE Trans. Biomed. Eng., vol. 46, no. 9, pp. 1150-1160, Sep. 1999.

[29] R. M. West, R. G. Aykroyd, S. Meng, and R. A. Williams, "Markov chain Monte Carlo techniques and spatial-temporal modeling for medical EIT," Physiological Meas., vol. 25, pp. 181-194, 2004 